Rev. Elev. Méd. vét. Pays trop., 1975, 28 (1) : 27-40

\title{
Données générales sur l'écologie de Glossina tachinoides Westwood, 1850, dans la réserve de Kalamaloué, vallée du bas-Chari
}

(I)

\author{
par J. GRUVEL $\left({ }^{*}\right)$
}

\begin{abstract}
RESUME
Après avoir présenté quelques aspects de la physiologie de Glossina tachinoides Westwood 1850 , l'auteur expose les résultats de ses observations sur l'écologie de cette espèce, étudiée dans une zone marginale de son aire de répartition: la Réserve de Kalamaloué dans la vallée du basChari. L'étude écologique envisage des relations de $G$. tachinoides avec le milieu environnant et aborde le problème de la dynamique de ses populations.
\end{abstract}

Glossina tachinoides est présente dans treize états africains qui sont d'Ouest en Est: la Guinée, le Mali, la Côte d'Ivoire, la HauteVolta, le Ghana, le Togo, le Dahomey, le Niger, le Nigéria, le Cameroun, le Tchad, la République centrafricaine et l'Ethiopie. Elle s'y trouve le plus souvent en compagnie de plusieurs autres espèces, mais se rencontre seule dans les régions les plus septentrionales. L'aire de répartition est discontinue au niveau du Soudan qui en est privé et se situe donc presque entièrement en Afrique occidentale et centrale. Elle y constitue une bande qui s'étend du $9^{\circ}$ au $20^{\mathrm{e}}$ degré de longitude Est sur une largeur variable, toujours comprise entre les $6^{e}$ et $14^{\mathrm{e}}$ parallèles Nord (fig. 1).

C'est au Tchad et en République centrafricaine que se situe la limite orientale de la répartition continue de cette espèce; limite incluse dans le bassin versant du lac Tchad. Elle n'existe pas dans le bassin adjacent du Congo. Dans ces deux Etats, sa distribution se

(*) Laboratoire de Farcha, B.P. 433 N'Djamena, République du Tchad. superpose au réseau hydrographique tracé par le Chari, ses affluents et ses défluents. Elle franchit ainsi le $12^{\mathrm{e}}$ parallèle, en aval de la capitale tchadienne, selon une bande étroite axée sur le cours inférieur du Chari à la frontière du Tchad et du Cameroun. C'est dans cette région, à la Réserve de Kalamaloué, que les études écologiques ont été poursuivies, pendant près de 6 ans (fig. 2).

\section{RECHERCHES SUR LA PHYSIOLOGIE DE G. TACHINOIDES}

Avant de décrire le milieu et de présenter les résultats des recherches sur l'écologie de G. tachinoides, il convient de rappeler quelques aspects de sa physiologie qui ont été envisagés plus particulièrement en rapport avec les phénomènes écologiques observés.

Ces recherches ont été pratiquées au laboratoire dans un élevage constitué à partir de pupes ou de glossines adultes provenant de la Réserve de Kalamaloué. La salle d'élevage, d'un volume de $15 \mathrm{~m}^{3}$ environ, était constam- 

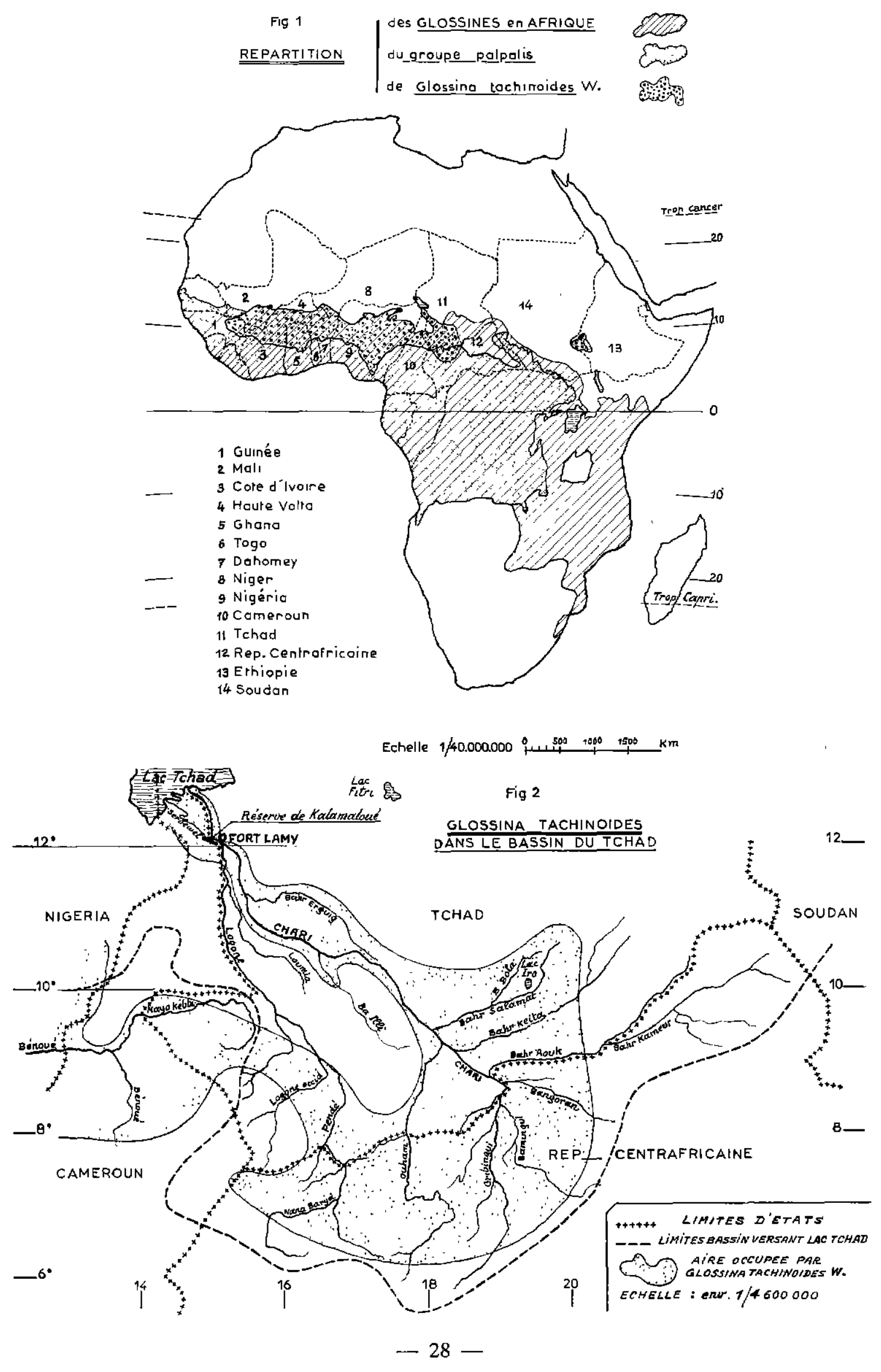
ment maintenue à la température de $25^{\circ}$, à une humidité oscillant entre $65-95$ p. 100 et soumise à des variations de lumière suivant celles du milieu extérieur. Les glossines étaient groupées en cages Roubaud et nourries quotidiennement sur oreilles de lapin.

Quelques observations complémentaires ont été effectuées parfois sur le terrain sur des mouches maintenues en cage.

Dans l'ensemble, ces recherches ont permis de retrouver pour $G$. tachinoides des lois physiologiques communes à toutes les espèces de glossines, mais également de préciser quelques points particuliers relatifs notamment aux fonctions de reproduction et de nutrition.

\section{REPRODUCTION}

Les relations établies pour diverses espèces, entre température et durée des cycles ovariens, périodes interlarvaires et pupaisons ont été confirmées pour $G$. tachinoides. Dans les conditions de l'élevage, ces durées sont respectivement de 40 jours, d'une semaine et de 30 jours en moyenne. Elles sont raccourcies par une élévation de température.

Les résultats particuliers qui ont pu être observés chez $G$. tachinoides concernent le cycle ovarien des femelles vierges, la larve libre, la pupaison et l'éclosion.

\section{Cycle ovarien chez les femelles vierges}

Normalement régulier chez les femelles fécondées au début de leur vie, le cycle ovarien présente chez les vierges des anomalies qui sont d'autant plus fréquentes que les femelles sont plus âgées. Ces anomalies sont le plus souvent représentées par une rétention des ovules mûrs dans les ovaires et les oviductes; rétention qui crée un obstacle mécanique à toute tentative d'insémination tardive. Ce n'est que chez les jeunes femelles, âgées de moins de 9 jours (chez lesquelles la $1^{\text {re }}$ ovulation n'a pas encore eu lieu) que les accouplements se révèlent constamment fécondants.

Ce type d'anomalies n'a pas été rencontré chez les femelles sauvages de $G$. tachinoides, ce qui suggère que, dans la nature, il n'y a pas de vierges âgées, vraisemblablement parce qu'elles sont toutes fécondées dès les premiers jours de leur vie.

\section{La larve libre}

Sitôt expulsée, la larve de $G$. tachinoides est très active et lorsque les conditions sont favorables, il lui faut moins d'un quart d'heure pour s'immobiliser et se transformer en pupe.

Les observations faites dans l'élevage du Laboratoire de Farcha ont montré que la durée de la vie larvaire libre est affectée par la lumière, l'humidité et la texture du sol. La larve fuit une lumière trop vive et recherche une humidité élevée; elle répond à ces deux conditions en s'enfonçant dans le sol et en s'immobilisant à quelques centimètres de profondeur, ne pénétrant pas les couches plus profondes trop humides et par là même trop compactes.

\section{Pupaison}

Le stade pupal de $G$. tachinoides se prolonge pendant une trentaine de jours dans les conditions de laboratoire. La durée de pupaison des pupes mâles est supérieure à celle des pupes femelles : 31 jours contre 28 . Elle varie également en raison inverse de la température dans des limites compatibles avec la survie de la nymphe, soit sensiblement entre 18 et 40 . Cette survie est surtout conditionnée par l'humidité dans laquelle baigne la pupe. Lorsqu'elle devient trop basse, le contenu de la pupe se dessèche et au laboratoire une humidité s'abaissant au-dessous de 40 p. 100 provoque une diminution du taux des éclosions par mortalité en pupe et la plupart des imagos qui atteignent leur terme ne sont pas viables.

A leur formation les pupes de $G$. tachinoides ont un poids moyen de $16,77 \mathrm{mg}$ avec des extrêmes de 10,5 et $22,5 \mathrm{mg}$. Il n'y a pas de différence significative entre les poids des pupes mâles et des pupes femelles qui diminuent parallèlement au cours de la pupaison. Celles dont le poids est inférieur à $10 \mathrm{mg}$ ont leur contenu altéré.

\section{Eclosion}

Les observations faites au laboratoire et sur le terrain montrent que les éclosions se font tôt le matin et en fin d'après-midi; cela en toute saison. Elles sont pratiquement nulles dans la mi-journée, aux heures les plus chaudes et les plus lumineuses. Il est remarquable de constater que les éclosions se font de préférence autour d'une heure moyenne optimals, le 
matin et l'après-midi, où les températures et luminosités correspondantes sont pratiquement identiques : de $22^{\circ}$ à $25^{\circ}$ et 1400 lux le matin, de $32^{\circ}$ et 500 Lux le soir aussi bien en saison fraîche qu'en saison chaude. Mais les difficultés qu'il y a à rattacher d'une manière rigoureuse les éclosions aux facteurs externes suggèrent que celles-ci pourraient représenter une forme de l'activité de $G$. tachinoides, dépendante de facteurs endogènes déterminant un rythme circadien propre.

\section{NUTRITION}

Comme les autres espèces de glossines, $G$. tachinoides montre une hématophagie stricte qui peut se manifester en élevage sur les hôtes les plus variés tels que : chèvres, ânes, porcs, cobayes, lapin et homme.

La quantité de sang absorbée par $G$. tachinoides est plus élevée pour les femelles que pour les mâles; en moyenne $11,92 \mathrm{mg}$ contre 7,39, soit 1,6 fois plus.

Les mâles s'alimentent plus irrégulièrement que les femelles qui prennent quotidiennement, dès leur fécondation, un repas copieux, variable cependant selon le moment de la période interlarvaire; très volumineux aussitôt après la ponte, plus faible et parfois nul avant celle-ci.

Les deux sexes ne résistent pas à un jeûne de plus de 48 heures. La digestion d'un repas normal dure 72 heures dans les conditions de laboratoire et la période d'inappétence persiste tant que le jabot et la partie antérieure de l'intestin moyen ne sont pas totalement vides, soit pendant une douzaine d'heures après le repas. Le cycle de la faim est court, compris entre 12 et 48 heures, aussi bien au laboratoire que dans la nature où sa brièveté a également pu être confirmée.

\section{RECHERCHES ECOLOGIQUES}

\section{ZONE D'ETUDE : RESERVE DE KALAMALOUE}

\section{Situation (fig. 3)}

Les études écologiques ont été faites, en toutes saisons à la Réserve de Kalamaloué.
Créée en 1948, cette Réserve s'étend sur la rive camerounaise du Chari sur une longueur d'environ $16 \mathrm{~km}$ et une largeur de 2 à $3 \mathrm{~km}$; sa surface est de près de 4000 ha.

\section{Climat (fig. 4)}

Elle est soumise à un climat sahélien typique. La saison des pluies s'étale sur près de 3 mois $1 / 2$, de juin à septembre, apportant pendant les années d'études une moyenne de $590 \mathrm{~mm}$ d'eau. Au cours de la saison sèche, s'observent les températures les plus basses et les plus élevées. Les minimums thermiques sont notés en général en décembre (extrêmes journaliers de $35^{\circ} 5$ et de $14^{\circ} 4$ ); les maximums en mai (variations journalières comprises entre $40^{\circ} 5$ et $22^{\circ}$. L'humidité varie régulièrement au cours de l'année entre son minimum atteint en février et son maximum en pleine saison des pluies. Les valeurs minimales de février varient entre 9 et 50 p. 100 , les maximales entre 58 et 96 p. 100 , en 24 heures.

Considérant les variations annuelles de la température et de l'humidité, l'année peut se diviser en 5 périodes caractéristiques:

1. la saison froide et sèche (novembre, décembre et janvier);

2. la saison chaude et sèche (février et mars);

3. la saison très chaude à humidité croissante (avril, mai);

4. la saison des pluies (juin, juillet, août et septembre);

5. Ia saison chaude à humidité décroissante (octobre).

Le vent est un facteur climatique important: de novembre à mars souffle l'Harmattan qui dessèche l'atmosphère et plus tard apparaissent les tornades brèves et violentes qui précèdent les pluies ou les accompagnent.

\section{3a Hydrographie (fig. 5)}

Le Chari et son défluent le Serbewel déterminent au niveau de la Réserve des conditions locales favorables au développement d'une végétation riveraine où peuvent vivre les glossines. Ces conditions sont cependant périodiquement modifiées par les variations de niveau des eaux libres qui présentent un écart très important entre les extrêmes et entraînent des inondations périodiques obligeant les glossines à changer d'habitat au cours de l'année. Une 
FIg 3 - SITUATION DE LA RÉSERVE DE KALAMALOUÉ

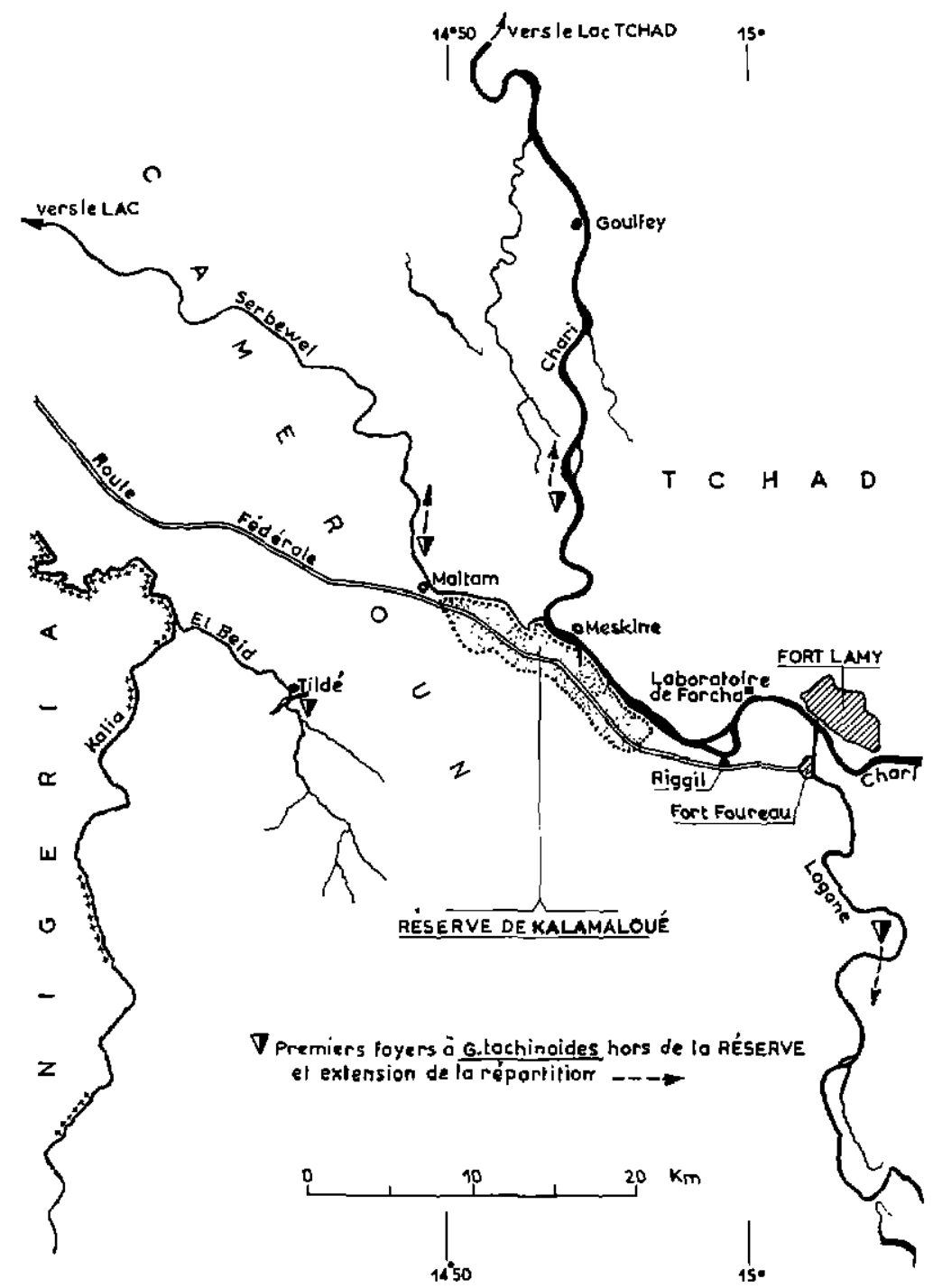

FIg 4 - MOYENHES DES TEMPERATURES ET TENSIONS DE VAPEUR SATURANTE Moyennes mensuelles des 4 derniēres onnées

(1) Solson iraiche

(2) 5 oison chaude sèche

(3) Sarsan Irós choude, humiditó en hausse

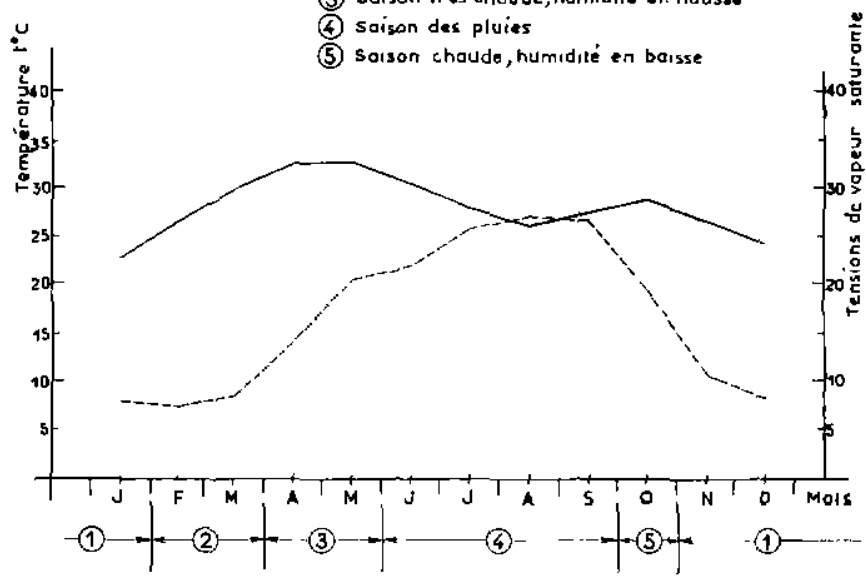




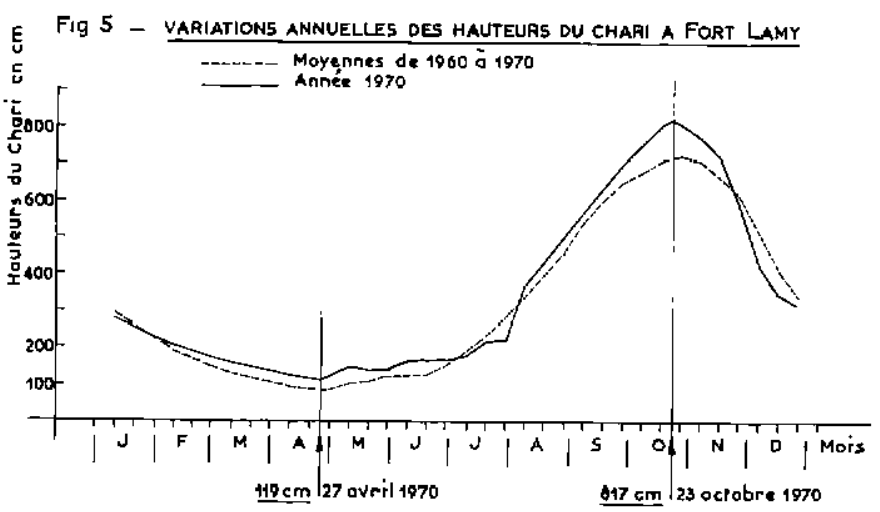

différence de $6,31 \mathrm{~m}$ et de 6 mois sépare le maximum de fin avril et le minimum de fin novembre. Le niveau reste bas la plus grande partie de l'année, inférieur à la valeur moyenne pendant près de 8 mois, de la mi-décembre à la mi-août. Les crues et les décrues sont rapides.

\section{Végétation (fig. 6)}

Les formations végétales arborées ou arbustives où vivent les glossines bordent les eaux libres des cours d'eau et sont désignées sous le nom de gîtes. Chaque gîte est déterminé par une espèce d'arbre dominante qui lui donne son aspect caractéristique et est installée selon le relief et la durée des inondations. Trois types de gîtes peuvent ainsi être distingués.
1. Gîtes exondés: parfois partiellement inondés par endroits aux périodes de très hautes eaux. Occupés pendant quelques semaines seulement par les glossines, ils sont composés de grands arbres, Diospyros mespiliformis, Tamarindus indica, Ziziphus mucronata et des lianes Paullinia pinnata auxquels se mêlent quelques Mitragyna inermis vers les parties basses atteintes par les eaux. Vers l'arrière pays les Acacia, Cadaba farinosa et Bauhinia rufescens leur succèdent. Le gîte $S_{1}$ répond à cette description.

2. Gîtes moyennement inondés : sont occupés par les glossines en périodes de crue et de décrue soit de 2 à 3 mois deux fois par an. Mitragyna inermis est l'espèce végétale dominante et caractérise le gîte $\mathrm{C}_{5}$.

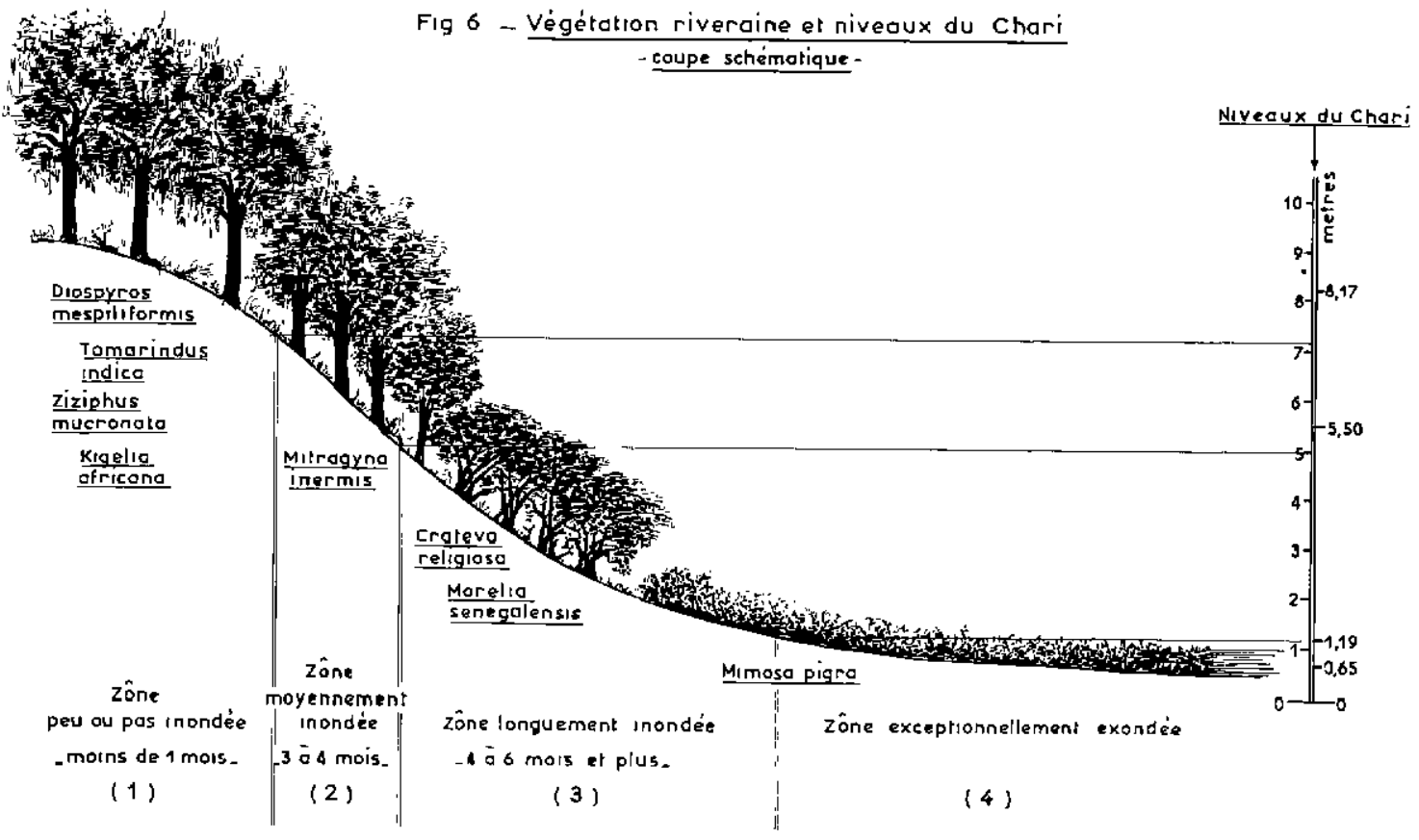




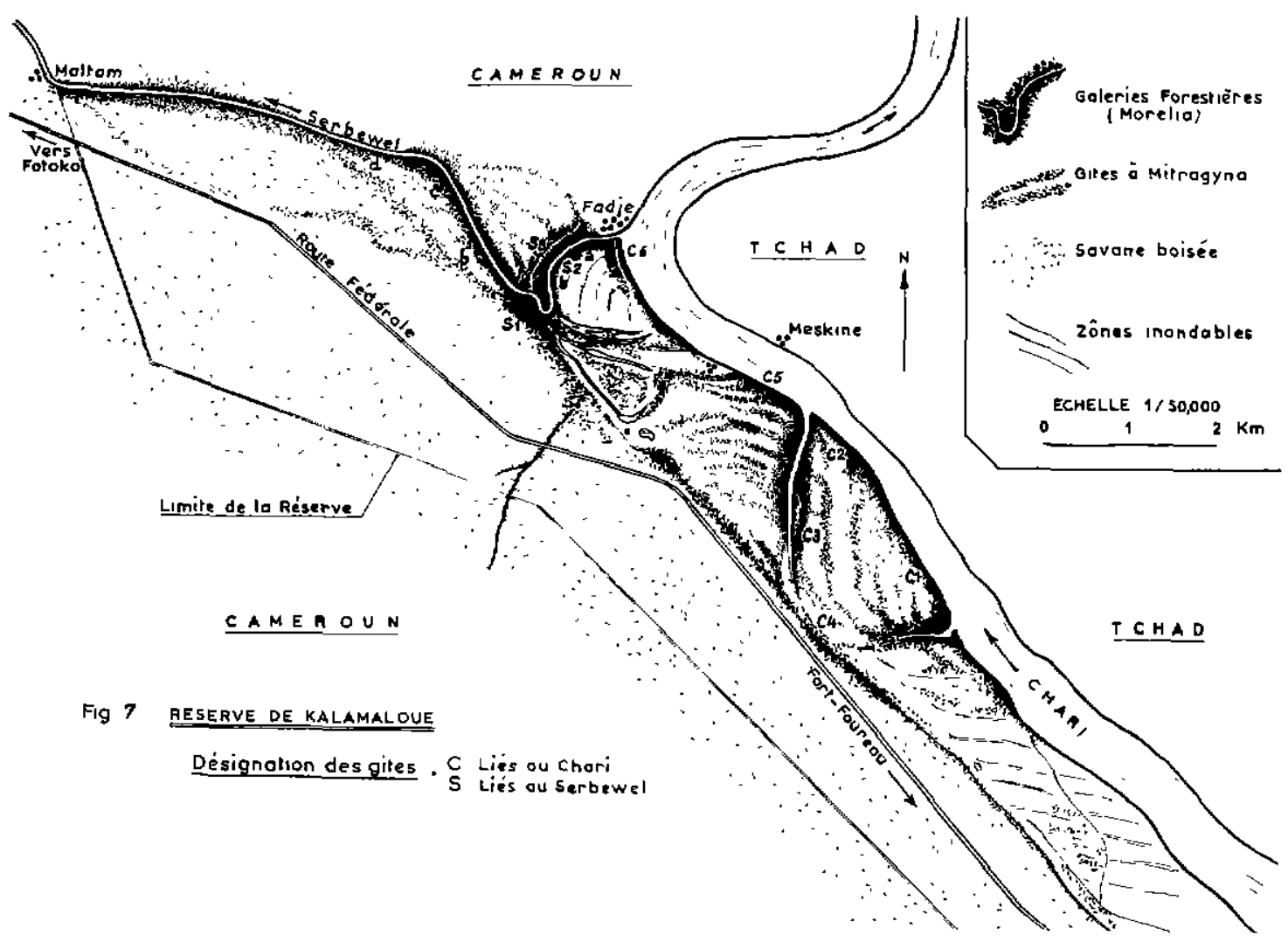

3. Gîtes très inondês : sont occupés pendant plus de 6 mois par $G$. tachinoides, de janvier à juillet et composés de Morelıa senegalensis avec quelques Crateva religiosa sur les parties hautes. Ils sont bordés vers les eaux libres de Mimosa pigra. Le gîte $\mathrm{C}_{3}$ est le plus typique de ces gîtes.

La distribution de ces gîtes $S_{1}, C_{5}$ et $C_{3}$ dans la Réserve est précisée sur la figure 7.

\section{5o Faune}

La Réserve de Kalamaloué possède une faune variée et abondante dont les représentants fréquentent les gîtes décrits.

\section{Vertébrés}

- Parmi les mammifères, les espèces les plus constantes sont les antilopes: cobs onctueux (Kobus defassa Rüppel), cobs de Buffon (Adenota kob Erxleben), cobs des roseaux (Redunca redunca Pallas), Guibs harnachés (Tragelaphus scriptus Pallas), céphalophes (Sylvicapra grammia L.). Les phacochères (Phacochaerus aethiopicus Pallas) et hippopotames
(Hippopotamus amphibius L.) sont également bien représentés.

Une mention toute particulière doit être faite en ce quí concerne les éléphants (Loxodonta africana Blumenbach). Habitants temporaires de la Réserve où on ne les observe que de janvier aux premières pluies (juin), ils s'y font rapidement remarquer par l'empreinte dont ils marquent le milieu. En effet, grands destructeurs, grands gaspilleurs, ces animaux dont le nombre croît chaque année, infligent des modifications importantes au biotope de quelques galeries ripicoles. Les arbres peu résistants tels que les Crateva sont, en certains endroits, tellement maltraités que de vastes clairières ont remplacé la voûte végétale protectrice. Ces destructions sont d'une importance capitale lorsqu'elles se font dans les formations végétales arborées denses qui constituent les gîtes à glossines de saison chaude. C'est là un facteur écologique de grand intérêt.

Vers le nord, la rive tchadienne est totalement nue sur de très grandes surfaces, victime des déboisements intenses qui se pratiquent, de plus en plus loin autour de N'Djamena. Au Nord-Ouest, entre Serbewel et Chari, une 
grande savane fait suite à la galerie bordant le Serbewel depuis son confluent.

Dans le décor de la végétation arborée proche des mares et des cours d'eau, on note l'activité des singes: singes verts (Cercopithecus aetiops tantalus Ogylby) et singes rouges (Erythrocebus patas Schreber); ces derniers, plus terrestres, s'enfoncent plus volontiers dans les hautes herbes des zones de savane.

A l'intérieur des galeries les plus sombres, des chauves-souris (Epomophorus gambianus Ogylby, Lavia frons E. Geoffroy, Nycteris aethiopica, Dobson) peuvent être observées dans leur vol silencieux.

A ces mammifères, dont la présence est facile à remarquer, il convient d'ajouter celle de nombreux carnivores plus discrets; les servals, genettes, mangoustes, civettes, hyènes sont fréquents alors que la panthère est rare.

- Les principaux reptiles sont:

- les crocodiles (Crocodylus niloticus Laurenti), difficiles à dénombrer, peu nombreux en apparence, restant localisés aux rives bordant les cours d'eau et mares permanentes;

- les varans : varan de terre (Varanus griseus Daudin), varan d'eau (V. niloticus L.) fréquemment au repos sous les mimosées de bordure ou sur les branches d'arbres surplombant l'eau des mares;

- les serpents : Naja haje L.; Bitis lachesis Laurenti et Python sebae Gmelin ne sont pas rares, quoique peu visibles.

- Bien qu'elle soit d'un moindre intérêt, on ne saurait passer sous silence la riche avifaune de la Réserve. Oiseaux sédentaires, migrateurs venus de très loin, sont toujours très nombreux et très riches en espèces; plus de 70 peuvent être dénombrées.

Par sa richesse, la faune de Kalamaloué constitue pour les glossines une exceptionnelle source de nourriture qui est un des facteurs fondamentaux de leur présence constante dans la Réserve.

\section{Invertébrés}

Les invertébrés sont très richement représentés à Kalamaloué. Seuls les Arthropodes connus pour jouer un rôle de prédateurs ou de parasites vis-à-vis des glossines adultes ont retenu l'attention. Les observations sont donc limitées aux Diptères Asilides et Bombylides (g. Thyridanthrax) et aux Araignées de la famille des Hersiilidae dont l'activité se révèle considérable à certaines périodes de l'année.

\section{Présence humaine}

La fréquentation humaine de la Réserve n'est pas négligeable:

- des villages de pêcheurs sont établis près de ses limites (Meskine, Fadjé) et même tolérés à l'intérieur; les habitants peuvent la traverser à pied par des sentiers traditionnels ou en pirogue en empruntant les bras morts ou les mares intérieures;

- des touristes viennent fréquemment la visiter en saison chaude.

L'homme constitue alors un appât sur lequel les glossines peuvent s'alimenter.

De plus, afin de faciliter l'observation des animaux, le Service des Eaux et Forêts du Cameroun fait annuellement brûler la savane. Ces feux ont une importance écologique sur laquelle nous reviendrons.

\section{Modifications observées à la Réserve et anx environs}

Les caractères d'isolement de la Réserve signalés plus haut, relatifs principalement à la présence des glossines, n'ont pas toujours été marqués aussi nettement qu'aujourd'hui; des changements notables aboutissent à en faire progressivement le seul îlot de végétation et de faune de la région :

\section{- A l'extérieur de la Réserve}

$\mathrm{Au}$ cours des dix dernières années toute la végétation ripicole qui s'étendait entre Riggil et la limite est de Kalamaloué, soit près de $5 \mathrm{~km}$ a été détruite. Cette zone est désormais cultivée. Il en est de même vers l'Ouest, le long du Serbewel, aux environs de Maltam, où la végétation dense riveraine s'amenuise peu à peu, se réduisant, lorsqu'elle existe encore, à une bande unique de Morelia senegalensis.

\section{- A l'intérieur de la Réserve}

A l'intérieur de ses limites, la Réserve a subi elle aussi quelques modifications dans le détail de ses massifs forestiers. Certains gîtes présentent une réduction de leur surface, due à l'ac- 
tion conjuguée des éléphants et des feux qui se répète chaque année en saison sèche. Une évolution se manifeste également au niveau des mares intérieures dont les plus importantes voient leurs rapports avec le Chari se modifier progressivement. De même à l'embouchure du Serbewel les bancs de sable s'étalent, s'allongent et réduisent considérablement la largeur du passage navigable en période de basses eaux.

\section{RESULTATS DES RECHERCHES ECOLOGIQUES}

En raison de la brève durée du stade larvaire libre, seules les pupes et les adultes de $G$. tachinoides, au repos et en activité, peuvent être régulièrement observées à Kalamaloué.

Les études présentées examinent successivement les relations des populations de G. tachinoides avec le milieu, leurs adaptations à ses variations et les conséquences d'une modification trop intense de celui-ci. Elles conduisent également à suivre l'évolution des populations au cours des saisons.

\section{Relations des glossines avec leur milieu}

Elles sont envisagées au niveau des pupes, puis des adultes au repos ou en activité.

\section{Les pupes}

Les pupes se rencontrent dans les parties des gîtes protégées par un couvert végétal qui réalise une ombre permanente, réduit la température et l'évaporation locale, là où le sol est ameubli par le passage ou le séjour prolongé d'animaux sauvages.

Les lieux de ponte sont toujours situés dans les gîtes occupés par les adultes, mais leur localisation varie au cours des saisons, en rapport avec les conditions thermiques et hygrométriques. Très dispersées pendant les pluies et au maximum des inondations, elles se concentrent en période sèche, dans des portions plus réduites des gîtes, sableuses ou argileuses. D'une manière générale, ces lieux de ponte suivent ou précèdent les mouvements de descente ou de montée des eaux et sont toujours rencontrées à proximité de celles-ci.

Les mesures de température et d'humidité pratiquées dans le sol au niveau des pupes de
G. tachinoides montrent qu'au cours de l'année, d'une saison fraîche à une saison chaude, la température varie de $19^{\circ}$ à $33^{\circ}$ et que $1^{\prime}$ humidité est toujours comprise entre 50 et 80 p. 100.

- Les variations thermiques entraînent des durées moyennes de pupaison de 23 à 38 jours; elles sont ainsi d'environ 1,6 fois plus longues en saison fraîche qu'en saison chaude.

- Les pupes récemment formées se trouvent là où l'humidité est la plus élevée, dans les endroits les plus abrités de l'insolation au moment de la ponte.

La pupaison, commencée dans une humidité élevée, s'achève généralement avant que celle-ci atteigne un taux inférieur à celui nécessaire à la survie des pupes qui est de 40 p. 100 (voir physiologie). Lorsque la décroissance naturelle de l'humidité au niveau des pupes atteint ce seuil critique, celles-ci subissent des altérations par dessèchement de leur contenu. C'est ce qui s'observe en saison chaude lorsque les pupes se forment en des endroits où l'humidité est trop basse, ou en toute saison lorsque les lieux de ponte sont bouleversés ou exposés à une insolation intense par destruction de la végétation protectrice, notamment après les passages des éléphants dans les gîtes.

Dans leur milieu naturel, les pupes peuvent être victimes de prédateurs et de parasites. A Kalamaloué, elles sont parfois parasitées par un Hyménoptère du genre Synthomosphyrum, mais surtout et beaucoup plus régulièrement par le Diptère Bombylide Thyridanthrax beckerianus. Ce dernier parasite s'observe constamment en saison chaude lorsque les lieux de ponte de G. tachinoides et des Thyridanthrax se rejoignent par l'action des conditions climatiques rigoureuses de l'époque.

\section{Les glossines au repos}

Les adultes au repos peuvent être, soit en état de somnolence sous l'effet de la digestion ou des conditions climatiques, soit en état de veille, à l'affût. Seuls les premiers, en état de repos complet, indifférents à toute sollicitation extérieure, sont étudiés ici.

Les observations ont été faites dès la fín de la saison fraîche et poursuivies pendant toute la saison chaude jusqu'à l'apparition des premières pluies, principalement sur les Morelia du gîte $\mathbf{C}_{3}$. 
Le repérage des $G$. tachinoides au repos est souvent difficile en raison de leur dispersion, mais surtout en raison de l'homochromie presque parfaite qui les unit à leur support végétal. Pour réduire cet inconvénient, nous avons préalablement marqué un grand nombre de mouches, différemment pour les mâles et les femelles par une tache de peinture ou par poudrage. Ces marques étant par ailleurs détectables la nuit par le faisceau d'une lampe à radiations ultra-violettes. Les lieux de repos, la hauteur des mouches ont ainsi pu être précisés et examinés en rapport avec les principaux facteurs climatiques (température, humidité, intensité lumineuse) dans le gîte ambiant et au niveau des points de repos. Ces observations ont été faites de jour et de nuit.

\section{Lieux de repos diurnes}

Ils sont constitués par les parties inférieures des troncs, les branches basses et les cavités des arbres, à l'abri du soleil et du vent.

Ils sont situés en bordure des gîtes, de leurs clairières ou des couloirs de passage des animaux; mais jamais au plus profond de la végétation. G. tachinoides s'y place toujours à la face inférieure des branches inclinées, la tête en position plus élevée que l'abdomen.

L'examen de $1849 G$. tachinoides des 2 sexes montre que :

a) à partir d'une température voisine de $31^{\circ}$ dans les gîtes, jusqu'au maximum enregistré de $41^{\circ}$, les hauteurs moyennes de repos diminuent régulièrement quand la température augmente (de $115 \mathrm{~cm}$ à $23 \mathrm{~cm}$ ). Aux températures inférieures à $30^{\circ}$ les glossines sont au repos, très dispersées, à des hauteurs variant de $\mathbf{1}$ à plus de $3 \mathrm{~m}$;

b) il n'y a pas de différence entre les hauteurs de repos des mâles et celles des femelles, quelle que soit la température;

c) lorsque la température du gîte est supérieure à $30^{\circ}$, celle au niveau des points de repos lui est toujours inférieure, mais sans jamais dépasser un maximum moyen de $33^{\circ}$;

d) l'humidité est liée naturellement à la température par une corrélation négative; les hauteurs de repos augmentent avec elle, mais à partir d'un minimum qui correspond à un taux d'humidité d'autant plus bas que la température est plus élevée; e) au niveau des points de repos, l'humidité est toujours supérieure à celle enregistrée dans le gîte ambiant, comprise entre 25 et 65 p. 100 selon l'endroit et l'heure de la journée;

f) l'intensité lumineuse perçue aux lieux de repos est presque toujours inférieure à $400 \mathrm{Lux}$, alors que dans le gîte elle peut atteindre, sans la dépasser, la valeur de 11000 Lux.

\section{Lieux de repos nocturnes}

Ils ont été recherchés en saison chaude, époque où les glossines quittent l'intérieur du gîte au crépuscule. Le pourcentage de $G$. tachinoides retrouvées au repos après leur marquage est faible (de l'ordre de 1 p. 100 sur 1896 mouches marquées quelques heures avant la recherche) et traduit une grande dispersion à l'extérieur du gîte où le soir la température est très inférieure à celle enregistrée à l'intérieur de celui-ci.

Les glossines pouvaient alors être retrouvées en bordure du gîte, sur le feuillage des Morelia ou sur les feuilles des plantes basses. La dispersion en surface et en hauteur de ces points de repos nocturnes est à rapprocher de celle observée le jour dans les gîtes lorsque la température est inférieure à $30^{\circ}$.

\section{Glossines en activité}

Les glossines en activité se manifestent rapidement à l'observateur par les attaques dont il est l'objet. G. tachinoides est fortement attirée par la présence de l'homme qui se révèle comme un excellent appât sur lequel il est possible de capturer les mouches qui viennent se poser. Cette propriété est à l'origine de la méthode d'échantillonnage qui a été employée. En effet, parmi toutes les techniques de captures d'adultes essayées : sur pièges fixes, sur animaux-appâts, sur captureurs eux-mêmes, seule cette dernière consistant à prélever au filet les glossines venant se poser sur les captureurs a été retenue. Elle est certes limitée à la seule fraction de la population en activité, sollicitée par la présence des captureurs, mais s'est montrée la plus adaptée aux études entreprises car applicable en tout lieu, en toute saison et permettant des captures nombreuses.

Les variations de l'activité journalière de G. tachinoides ont été appréciées par le nombre de captures enregistrées, du lever au coucher du soleil, pendant 30 minutes autour de chaque 
heure de la journée; chaque demi-heure de travail constituant une séance de capture et étant caractérisée par le nombre de glossines capturées, la température, l'humidité et l'intensité lumineuse au lieu même de l'action des captureurs. L'activité de 33000 glossines a été ainsi appréciée.

Les diagrammes représentant les variations de l'activité journalière des $G$. tachinoides étudiées en un même lieu pendant plusieurs jours consécutifs ne sont pas superposables et les niveaux d'activité, différents aux mêmes heures de ces différents jours, montrent que $G$. tachinoides ne se manifeste que sous l'effet de certaines conditions extérieures dont l'intensité d'action agit sur le nombre de glossines sollicitées. On peut ainsi noter que :

- les activités ne s'observent qu'entre $18^{\circ}$ $40^{\circ} \mathrm{C}$ et $0,7-11000 \mathrm{Lux}$;

- les représentations graphiques des activités horaires journalières étudiées au cours d'un cycle annuel montrent que les courbes ont un profil différent selon les périodes de l'année telles qu'elles ont été définies antérieurement. Chacune d'elle se caractérise par l'un des trois types d'activité suivants :

1. à un seul «pic » dans la mi-journée; en saison fraîche 1 , en fin de la $2^{\mathrm{e}}$ saison chaude 5;

2. à deux "pics ", situés le matin et le soir, séparés par une très forte réduction ou une annulation totale des activités pendant la mi-journée en saison très chaude 3 ;

3. avec un plateau étalé dans la journée aux saisons intermédiaires 2 et 4 (saison des pluies).

- Ces variations saisonnières ont conduit à considérer l'influence des différents facteurs climatiques fondamentaux sur l'activité de $G$. tachinoides. L'étude de leurs actions simultanées n'est pas accessible par l'application de méthodes statistiques simples; par contre, il est possible d'examiner l'effet de ces facteurs considérés isolément, puis deux à deux.

\section{Considérés isolément}

a) jusqu'à une température comprise dans la classe des $32^{\circ} 5$ (31.5 à $\left.33^{\circ} 5\right)$, l'activité augmente; elle diminue ensuite lorsque la température croît au-delà de cette valeur;

b) l'activité de $G$. tachinoides apparaît plus intense aux humidités voisines de 65 p. 100 ; c) elle augmente avec l'intensité lumineuse jusqu'à ce que celle-ci atteigne 11000 Lux; elle diminue très rapidement au-delà.

\section{Considérés deux à deux}

Il faut d'abord noter que l'examen des températures, intensités lumineuses et humidités correspondant à 59 maximums d'activité observés à différents moments d'une même année montre que ces maximums ont toujours lieu à une température de $31^{\circ} 06 \pm 0,72$ et sous une intensité lumineuse également sensiblement constante, de l'ordre de $2500 \mathrm{Lux}$ (correspondant à l'indice $14,88 \pm 0,82$ de la cellule photométrique utilisée). Dans les conditions de ces observations, les maximums d'activité se révèlent indépendants de l'humidité.

Pour cette raison, n'est retenue que l'action simultanée des deux facteurs température et intensité lumineuse. L'examen montre que les maximums d'activité se placent à des intensités lumineuses d'autant plus faibles que la température s'éloigne, en diminuant ou en augmentant, des valeurs de la classe des $30^{\circ} 5$ $\left(29^{\circ} 5\right.$ à $\left.31^{\circ} 5\right)$. Ce fait traduit des inversions du phototropisme manifestées par les glossines selon les conditions thermo-lumineuses dans lesquelles elles se trouvent.

Les effets de l'association des facteurs thermiques et lumineux sont particulièrement intéressants car ils expliquent, dans une large mesure, les moments et les lieux où se manifeste l'activité. Ils rendent compte de la nature des déplacements que peuvent effectuer les glossines.

\section{Déplacements}

$G$. tachinoides change constamment de lieux d'activité. Elle se déplace par ses propres moyens ou se laisse entraîner par un véhicule, un animal ou un homme sur lequel elle se pose temporairement. Elle se disperse ainsi à l'intérieur ou à l'extérieur des gîtes qu'elle fréquente normalement.

Les dispersions à l'intérieur des gîtes se font durant la joumée en saison fraîche ou intermédiaire, le matin et le soir en saison chaude, lorsque la température et la lumière locales sont comprises dans les limites précisées précédemment. En vol, elles peuvent parcourir $1 \mathrm{~km}$ par jour selon les trajets dégagés des sous-bois. Aux heures chaudes, G. tachinoides 
baisse d'activité et s'oriente vers les endroits les plus ombragés pour se placer au repos (2 sexes) ou pour aller pondre (femelles en fin de gestation); c'est en effet au début de l'après-midi que les pontes sont déposées le plus fréquemment.

Les dispersions à l'extérieur des gîtes s'observent lorsque la température et l'intensité lumineuse sont dans les limites favorables. C'est le cas en saison chaude où les glossines sortent des gîtes très tôt le matin et très tard le soir, trouvant leurs lieux de repos nocturnes hors de ceux-ci. C'est le cas également en saison des pluies, pendant la journée, lorsque les pluies ont abaissé la température et lorsque la nébulosité réduit l'intensité lumineuse. C'est à l'occasion de ces dispersions extérieures que les glossines qui ne regagnent pas obligatoirement leur gîte de départ peuvent être amenées à changer d'habitat plusieurs fois dans l'année.

\section{Repas}

Par leurs déplacements effectués à l'intérieur ou à l'extérieur des gîtes, les $G$. tachinoides affamées pourront se poser sur un animal qui les attire et y prélever leur repas de sang.

La recherche de l'origine du sang absorbé par les glossines se fait par les tests sérologiques classiques appliqués au contenu digestif des mouches récemment gorgées, capturées au repos.

1154 prélèvements effectués pendant la saison chaude dans le gîte $C_{3}$, révèlent que $8 \mathrm{p}$. 100 des repas de $G$. tachinoides sont pris sur des reptiles (varan), le reste sur des mammifères : primates (homme; 5,63 p. 100), suidés (phacochères; 12,21 p. 100) et bovidés (72,18 p. 100$)$. Parmi ceux-ci, le guib harnaché intervient dans 86,09 p. 100 des repas.

Ces analyses montrent par ailleurs que les mâles et les femelles se comportent de la même façon vis-à-vis des hôtes cités.

Cette liste des hôtes nourriciers est tout à fait comparable à celle des espèces constamment présentes dans les gîtes de saison chaude. Varans, phacochères, guibs, se tiennent normalement dans les mêmes buissons que ceux qui abritent les glossines et les pêcheurs se déplacent sur les mares résiduelles qui les bordent. Le cobs de buffon et cobs defassa, plus rarement piqués par les mouches se tien- nent effectivement loin des gîtes et les quelques repas pris sur eux résultent d'une rencontre du soir ou du matin lorsqu'ils viennent s'abreuver aux points d'eau. Ces résultats tendent à montrer que $G$. tachinoides adopte facilement l'hôte qui se présente.

\section{2o Dynamique des populations}

L'étude de la dynamique des populations ne peut être abordée valablement que par l'observation des adultes. Elle a été envisagée du point de vue des rapports des sexes, de leur âge et de leur densité.

\section{Rapport des sexes}

Exprimé par le pourcentage des femelles, il a été estimé à l'éclosion, parmi les captures d'adultes en activité ou au repos.

- A l'éclosion, il est statistiquement égal à l'unité (à une approximation de 95 p. 100 sur 11681 observations).

- Parmi les G. tachinoides capturées en activité, le pourcentage de femelles est, pour l'ensemble de l'année et des gîtes prospectés, de l'ordre de 33 p. 100 . Il ne s'en écarte qu'en mai où il s'abaisse à 18 p. 100 et en octobre où il atteint un maximum de 48,50 p. 100 .

Le pourcentage le plus bas s'observe lorsque les glossines sont concentrées au gîte $\mathrm{C}_{3}$ en saison chaude et que leurs déplacements sont faibles. Par contre les pourcentages élevés de fin de saison des pluies, voisins de 50 p. 100 , s'observent aux périodes de l'année où $G$, tachinoides change d'habitat, effectuant ses migrations saisonnières dans la réserve, les femelles manifestant une intense activité.

- Parmi les G. tachinoides capturées au repos, dont 3500 individus ont été observés au gîte $\mathrm{C}_{3}$, le pourcentage de femelles est toujours plus élevé que parmi les glossines en activité, dans les mêmes lieux et aux mêmes moments. Aux heures les plus chaudes des mois les plus chauds, lorsque les activités deviennent nulles, le pourcentage de femelles au repos atteint la valeur de 50 p. 100 . Cette valeur, observée chez $G$. tachinoides aux périodes de migrations et chez celles au repos alors que l'activité est nulle, suggère qu'elle pourrait représenter la proportion réelle des femelles dans les populations naturelles. 


\section{Age}

Les essais de détermination de l'âge des G. tachinoides ne se sont révélés fructueux que pour les femelles, par application de la méthode d'estimation de l'âge physiologique. En tenant compte de la variation de durée du cycle ovarien avec la température ambiante, il est possible d'évaluer l'âge moyen d'un échantillon. Il apparaît alors que la longévité varie en raison inverse de la température : de l'ordre de 1 mois en saison chaude et de 3 mois en saison fraîche.

\section{Densité des populations}

Les résultats sur l'estimation des densités des populations de $G$. tachinoides ont été obtenus par l'application de deux méthodes : celle des captures horaires et celle des captures-recaptures.

- Les moyennes des captures horaires journalières expriment la densité apparente. Dans les gîtes où les glossines sont présentes d'une façon continue pendant plusieurs mois, elle est maximale vers le milieu de la période d'occupation par les mouches, soit en mai au gîte $\mathrm{C}_{3}$ lorsque $G$. tachinoides se concentre dans les portions habitables réduites du gîte, soit en octobre au gîte $S_{1}$ qui constitue à cette époque l'un des rares habitats favorables aux glossines.

- La méthode des captures-recaptures permet d'approcher la densité réelle des populations. Elle n'est acceptable que lorsque le nombre de mouches marquées recapturées après un délai de 48 heures est suffisamment grand; ce qui n'a pas toujours pu être réalisé au cours des observations et traduit la faiblesse de cette méthode d'investigation par les populations de glossines.

Les deux méthodes s'accordent pour mettre en évidence deux maximums annuels qui se placent aux mêmes saisons, mais ne sont pas exactement superposables. Dans les conditions de ces observations, il n'apparaît pas de rapport constant entre densité réelle et densité apparente. Celle-ci diminue quand les populations se concentrent dans quelques portions d'un gîte antérieurement occupé en totalité.

La méthode de captures-recaptures a permis à cette époque (mois d'avril-mai) d'estimer la population réelle des mâles dans le gîte $\mathrm{C}_{3}$ à une densité de l'ordre de 500 par hectare. Appliquée aux deux sexes, cette méthode a montré que le pourcentage des femelles était toujours supérieur à celui noté dans les simples captures et souvent voisin de 50 p. 100.

Les fluctuations qui viennent d'être signalées dans les effectifs des populations de G. tachinoides à Kalamaloué résultent du potentiel reproducteur des femelles, de leur longévité et des durées de pupaison.

- Il a déjà été noté que le cycle ovarien, la gestation et la pupaison étaient accélérés par une augmentation de température: augmentation qui réduit la longévité. Il semble bien que seules les variations de durée des pupaisons ont un retentissement sur la population générale, laugmentant lorsque la température croît, la réduisant lorsqu'elle diminue.

- L'association de températures élevées et de très basses humidités détermine de mars à juin, mais surtout en avril et mai, la période la plus critique de l'année. Dans les gîtes fréquentés, c'est l'époque où les populations amorcent une sensible diminution due à des mortalités d'adultes, à la prédation par des Asilides ou par des Araignées, mais surtout à une baisse du taux des éclosions. Celle-ci résulte de l'action conjointe de la mortalité en pupes par dessiccation (qui peut atteindre 30 p. 100 de celles examinées) et du parasitisme intense exercé à cette époque par Thyridanthrax beckerianus (pouvant affecter plus de 20 p. 100 des pupes récoltées). Seulement un peu plus de 50 p. 100 des pupes observées sont alors capables de fournir des adultes viables.

Ces effets réducteurs des facteurs climatiques ou biotiques sur les populations de $G$. tachinoides ne s'atténuent qu'à l'installation de la saison des pluies qui abaisse la température et élève l'hygrométrie. Mais il faut remarquer que là encore les taux d'éclosion peuvent être affectés par l'immersion des pupes lors des fortes pluies ou des inondations.

\section{CONCLUSION}

Les études sur la physiologie de G. tachinoides montrent que cette espèce obéit à des lois physiologiques tout à fait comparables à celles d'autres espèces, notamment en ce qui concerne la reproduction. Elle est par contre plus exigeante que beaucoup d'autres en ce qui concerne les prises de repas qui répondent à un cycle de la faim relativement court. 
Les études sur le comportement de G. tachinoides montrent que la réalisation de ses rythmes physiologiques ne peut se faire que dans des conditions très étroites. En effet elle ne peut survivre que sous le couvert végétal dense où elle trouve les conditions convenables de température, humidité et lumière. Volant peu, seuls ses lieux de repos constituent en fait son véritable milieu de vie dont elle ne s'écarte en période d'activité que dans des limites restreintes. Sous ce couvert végétal se maintiennent également les hôtes nourriciers sur lesquels elle peut se nourrir fréquemment ainsi que l'exige sa physiologie digestive.

Toutes ces conditions sont réalisées dans les massifs forestiers de la vallée du bas Chari, à Kalamaloué en bordure du fleuve ou des mares permanentes. G. tachinoides ne s'éloigne guère de la proximité des eaux libres et abandonne très rapidement après la décrue les gîtes devenus trop arides. Cette proximité de l'eau libre constitue un élément fondamental de son écologie dans ces régions septentrionales à climat sévère, car elle permet le maintien d'une végétation riveraine dense et celui de la faune sauvage. La preuve en est donnée actuellement par la sécheresse qui sévit dans ces régions et qui, en asséchant les points d'eau habituels, entraîne le flétrissement de la végétation qui réduit considérablement toute possibilité de survie aux glossines et au gibier local (*).

La connaissance des lieux de repos, des rythmes d'activité, des déplacements et des densités des populations des glossines sont à l'origine de la préparation de programmes de recherches et d'action dans la lutte contre l'un des principaux vecteurs des trypanosomoses africaines.

(*) J. GRUVEL. Sécheresse, écologie et lutte contre $G$. tachinoides dans la vallée du Bas-Chari. Rev. Elev. Méd. vét. Pays trop., 1974, 27 (4):

\section{SUMMARY}

\section{General data on the ecology of Glossina tachinoides Westwood 1850} in the Kalamaloué reserve, Lower Chari River Valley

After describing some aspects of G. tachinoides Westwood 1850 physiology, the author reports the results of his observations on the species ecology, which was studied in a marginal zone of its distribution area, the Kalamaloue reserve in the Lower Chari River Valley. In this ecological study, the relationship between $G$. tachinoides and its environment is discussed and the problem of population dynamics is tackled.

\section{RESUMEN}

Datas generales sobre la ecologia de Glossina tachinoides Westwood 1850, en el coto de Kalamalue, valle del bajo Chari

El autor presenta algunos aspectos de la fisiologia de $G$. tachinoides Westwood 1850 y expone los resultados de sus observaciones sobre la ecologia de dicha especie, estudiada en una parte marginal de su zona de repartición: el coto de Kalamalue en la valle del bajo Chari.

Desde el punto de vista ecologico, se estudian las relaciones de $G$. tachinoides con el medio ambiente y el problema de la dinámica de estas poblaciones. 